\title{
Rhodium-Catalyzed Reactions of Dithiols and 1,4-Bis(bromomethyl)benzenes Leading To Enantioenriched Dithiaparacyclophanes
}

\author{
Ken Tanaka,* Tomoko Hori, Takuya Osaka, Keiichi Noguchi, and Masao Hirano \\ Department of Applied Chemistry, Graduate School of Engineering, and Instrumentation \\ Analysis Center, Tokyo University of Agriculture and Technology, Koganei, Tokyo \\ 184-8588, Japan
}

\section{General}

Anhydrous THF (No. 186562) and $\mathrm{Et}_{3} \mathrm{~N}$ (No. 471283) were obtained from Aldrich and used as received. All other reagents were obtained from commercial sources and used as received. All reactions were carried out under an atmosphere of $\mathrm{Ar}$ or $\mathrm{N}_{2}$ in oven-dried glassware with magnetic stirring. Dibromides $\mathbf{2} \mathbf{a}^{1}$ and $\mathbf{2} \mathbf{d}^{2}$ were prepared according to the literatures.

\section{Synthesis of Dibromides}

\section{1,4-Bis(bromomethyl)-2,5-diethylbenzene (2b).}<smiles>CCc1cc(CBr)c(CC)cc1CBr</smiles>

A $33 \%$ solution of $\mathrm{HBr}$ in glacial acetic acid $(33.2 \mathrm{~mL}, 192.22 \mathrm{mmol})$ was added dropwise to a suspension of 1,4-diethylbenzene $(4.30 \mathrm{~g}, 32.0 \mathrm{mmol})$ and paraformaldehyde (2.21 g, $73.7 \mathrm{mmol})$. The reaction mixture was refluxed for $18 \mathrm{~h}$, during which $\mathrm{HBr}$ in glacial acetic acid $(3.0 \mathrm{~mL}, 18.0 \mathrm{mmol})$ was added twice at $6 \mathrm{~h}$ intervals. The resulting mixture was cooled to room temperature, diluted with water $(100 \mathrm{~mL})$, and extracted with ethyl acetate $(100 \mathrm{~mL})$. The organic phase was neutralized with saturated aqueous $\mathrm{NaHCO}_{3}$, dried $\left(\mathrm{Na}_{2} \mathrm{SO}_{4}\right)$, concentrated under vacuum, and purified by column chromatography on silica gel (hexane/EtOAc = 50:1), which furnished $\mathbf{2 b}(4.29 \mathrm{~g}, 13.40 \mathrm{mmol}, 42 \%$ yield). Colorless solid; Mp 89-90 ${ }^{\circ} \mathrm{C}$; IR (KBr) 2960, 2876, 1459, 1208, $906 \mathrm{~cm}^{-1}$; ${ }^{1} \mathrm{H}$ NMR $\left(\mathrm{CDCl}_{3}, 300 \mathrm{MHz}, \delta 7.16(\mathrm{~s}, 2 \mathrm{H}), 4.50(\mathrm{~s}, 4 \mathrm{H}), 2.73(\mathrm{q}, J=5.4 \mathrm{~Hz}, 4 \mathrm{H}), 1.28(\mathrm{t}, J=5.4 \mathrm{~Hz}\right.$, $6 \mathrm{H}) ;{ }^{13} \mathrm{C} \mathrm{NMR}\left(\mathrm{CDCl}_{3}, 75 \mathrm{MHz}\right) \delta 140.8,135.9,130.9,31.3,24.6,14.8$; HRMS (FAB) calcd for $\mathrm{C}_{12} \mathrm{H}_{17} \mathrm{Br}_{2}[\mathrm{M}+\mathrm{H}]^{+}$318.9697, found 318.9665 .

\section{1,4-Bis(bromomethyl)-2,5-diisopropylbenzene (2c).}

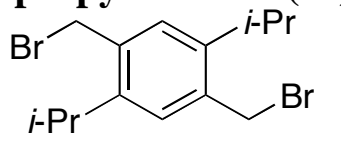


A $33 \%$ solution of $\mathrm{HBr}$ in glacial acetic acid $(3.00 \mathrm{~mL}, 18.0 \mathrm{mmol})$ was added dropwise to a suspension of 1,4-diisopropylbenzene $(0.487 \mathrm{~g}, 3.0 \mathrm{mmol})$ and paraformaldehyde (0.360 g, $12.0 \mathrm{mmol})$. The reaction mixture was refluxed for $42 \mathrm{~h}$, during which $\mathrm{HBr}$ in glacial acetic acid $(3.0 \mathrm{~mL}, 18.0 \mathrm{mmol})$ was added five times at $8 \mathrm{~h}$ intervals. The resulting mixture was cooled to room temperature, diluted with water $(50 \mathrm{~mL})$, and extracted with ethyl acetate $(50 \mathrm{~mL})$. The organic phase was neutralized with saturated aqueous $\mathrm{NaHCO}_{3}$, dried $\left(\mathrm{Na}_{2} \mathrm{SO}_{4}\right)$, and concentrated under vacuum. Recrystallization from $\mathrm{CH}_{2} \mathrm{Cl}_{2} /$ hexane gave 2c (0.546 g, $1.57 \mathrm{mmol}, 52 \%$ yield). Colorless solid; Mp $126{ }^{\circ} \mathrm{C}$; IR (KBr) 2966, 2927 , 2868, 2360, $1213 \mathrm{~cm}^{-1} ;{ }^{1} \mathrm{H} \mathrm{NMR}\left(\mathrm{CDCl}_{3}, 300 \mathrm{MHz}\right.$,) $\delta 7.21$ (s, 2H), 4.54 (s, 4H), 3.24 (sept, $J=6.9 \mathrm{~Hz}, 2 \mathrm{H}), 1.28(\mathrm{~d}, J=6.9 \mathrm{~Hz}, 12 \mathrm{H}) ;{ }^{13} \mathrm{C} \mathrm{NMR}\left(\mathrm{CDCl}_{3}, 75 \mathrm{MHz}\right) \delta 145.5,135.4$, 128.2, 31.5, 28.5, 23.8; HRMS (FAB) calcd for $\mathrm{C}_{14} \mathrm{H}_{20} \mathrm{Br}_{2}[\mathrm{M}]^{+}$345.9932, found 345.9969.

\section{Synthesis of Dithiaparacyclophanes}

General Procedure using $\mathbf{R h C l}\left(\mathrm{PPh}_{3}\right)_{3}$ Catalyst (Table 1, Entry 1): A THF (2.0 mL) solution of 1,5-pentanedithiol (1a, $34.1 \mathrm{mg}, 0.25 \mathrm{mmol}$ ) and 1,4-bis(bromomethyl)-2,5 -dimethylbenzene (2a, $50.8 \mathrm{mg}, 0.25 \mathrm{mmol}$ ) was added to a THF (2.2 mL) solution of $\mathrm{RhCl}\left(\mathrm{PPh}_{3}\right)_{3}(11.6 \mathrm{mg}, 0.0125 \mathrm{mmol})$. Then, $\mathrm{Et}_{3} \mathrm{~N}(0.80 \mathrm{~mL})$ was added to the mixture. The mixture was stirred at $\mathrm{rt}$ for $16 \mathrm{~h}$. The resulting mixture was concentrated and purified by preparative TLC (hexane/EtOAc $=10: 1)$, which furnished 3aa $(33.2 \mathrm{mg}, 0.498 \mathrm{mmol}, 50 \%$ yield) as a colorless solid.

General Procedure using $\left[\mathrm{Rh}(\operatorname{cod})_{2}\right] \mathrm{BF}_{4} /(S)$-BINAPHANE Catalyst (Table 2, Entry 1): A THF $(1.0 \mathrm{~mL})$ solution of $(S)$-BINAPHANE $(17.5 \mathrm{mg}, 0.025 \mathrm{mmol})$ was added to a THF $(1.0 \mathrm{~mL})$ solution of $\left[\mathrm{Rh}(\operatorname{cod})_{2}\right] \mathrm{BF}_{4}(10.2 \mathrm{mg}, 0.025 \mathrm{mmol})$. The mixture was stirred at $\mathrm{rt}$ for $1 \mathrm{~h}$. To the mixture was added a THF (2.2 mL) solution of 1,5-pentanedithiol (1a, $34.1 \mathrm{mg}, \quad 0.25 \mathrm{mmol}$ ) and 1,4-bis(bromomethyl)-2,5 -dimethylbenzene $(\mathbf{2 a}, 73.0 \mathrm{mg}, 0.25 \mathrm{mmol})$. Then, $\mathrm{Et}_{3} \mathrm{~N}(0.8 \mathrm{~mL})$ was added to the mixture. The mixture was stirred at $\mathrm{rt}$ for $16 \mathrm{~h}$. The resulting mixture was concentrated and purified by preparative TLC (hexane/EtOAc $=10: 1)$, which furnished $(-)-3 a a(29.7 \mathrm{mg}, 0.45 \mathrm{mmol}$, $45 \%$ yield, $49 \%$ ee) as a colorless solid.

\section{(-)-11,14-Dimethyl-2,8-dithia[9]paracyclophane [(-)-3aa].}

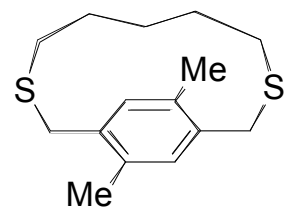

Colorless solid; Mp $190-191{ }^{\circ} \mathrm{C}$; $[\alpha]^{25}{ }_{\mathrm{D}}-50.3^{\circ}\left(\mathrm{CHCl}_{3}, c 1.330,49 \%\right.$ ee); IR (KBr) 2919, 2843, 1455, $1437 \mathrm{~cm}^{-1} ;{ }^{1} \mathrm{H}$ NMR $\left(\mathrm{CDCl}_{3}, 300 \mathrm{MHz}\right) \delta 6.86(\mathrm{~s}, 2 \mathrm{H}), 3.81(\mathrm{~d}, J=12.9$ $\mathrm{Hz}, 2 \mathrm{H}), 3.63(\mathrm{~d}, J=12.9 \mathrm{~Hz}, 2 \mathrm{H}), 2.52-2.41(\mathrm{~m}, 2 \mathrm{H}), 2.44(\mathrm{~s}, 6 \mathrm{H}), 1.86(\mathrm{dt}, J=13.8,8.1$ $\mathrm{Hz}, 2 \mathrm{H}$ ), 0.96 (sept, $J=6.6 \mathrm{~Hz}, 2 \mathrm{H}$ ), 0.74 (quint, $J=6.9 \mathrm{~Hz}, 2 \mathrm{H}$ ), 0.34 (sept, $J=6.6 \mathrm{~Hz}$, $2 \mathrm{H}) ;{ }^{13} \mathrm{C} \mathrm{NMR}\left(\mathrm{CDCl}_{3} 75 \mathrm{MHz}\right) \delta 134.4,134.0,133.3,34.9,29.9,27.7,24.5,18.4$; HRMS 
(FAB) calcd for $\mathrm{C}_{15} \mathrm{H}_{23} \mathrm{~S}_{2}[\mathrm{M}+\mathrm{H}]^{+}$267.1241, found 267.1200; CHIRALCEL OD-H, hexane $/ 2-\mathrm{PrOH}=90: 10,1.0 \mathrm{~mL} / \mathrm{min}$, retention times: $9.5 \mathrm{~min}$ (major isomer) and $24.2 \mathrm{~min}$ (minor isomer).

\section{(-)-11,14-Diethyl-2,9-dithia[10]paracyclophane [(-)-3ab].}

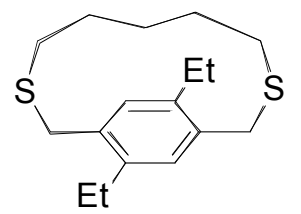

Colorless oil; $[\alpha]^{25}-67.2^{\circ}\left(\mathrm{CHCl}_{3}, c 1.730,36 \%\right.$ ee); IR (neat) 2961, 2923, 2870, 1499, $1453 \mathrm{~cm}^{-1} ;{ }^{1} \mathrm{H} \mathrm{NMR}\left(\mathrm{CDCl}_{3}, 300 \mathrm{MHz}\right) \delta 6.92(\mathrm{~s}, 2 \mathrm{H}), 3.89(\mathrm{~d}, J=12.9 \mathrm{~Hz}, 2 \mathrm{H}), 3.65(\mathrm{~d}, J$ $=12.9 \mathrm{~Hz}, 2 \mathrm{H}), 2.97-2.74(\mathrm{~m}, 4 \mathrm{H}), 2.50-2.41(\mathrm{~m}, 2 \mathrm{H}), 1.87(\mathrm{dt}, J=7.8,13.8 \mathrm{~Hz}, 2 \mathrm{H}), 1.28$ $(\mathrm{t}, J=7.5 \mathrm{~Hz}, 6 \mathrm{H}), 0.92$ (sept, $J=6.9 \mathrm{~Hz}, 2 \mathrm{H}$ ), 0.74 (quint, $J=6.6 \mathrm{~Hz}, 2 \mathrm{H}$ ), 0.41 (sept, $J=$ $6.6 \mathrm{~Hz}, 2 \mathrm{H}) ;{ }^{13} \mathrm{C} \mathrm{NMR}\left(\mathrm{CDCl}_{3}, 75 \mathrm{MHz}\right) \delta 139.8,134.1,131.6,34.6,30.0,27.7,24.6,24.4$, 14.6; HRMS (EI) calcd for $\mathrm{C}_{17} \mathrm{H}_{26} \mathrm{~S}_{2}[\mathrm{M}]^{+}$294.1476, found 294.1495; CHIRALCEL OD-H, hexane $/ 2-\mathrm{PrOH}=97: 3,0.8 \mathrm{~mL} / \mathrm{min}$, retention times: $5.5 \mathrm{~min}$ (major isomer) and $9.0 \mathrm{~min}$ (minor isomer).

(-)-11,14-Diisopropyl-2,9-dithia[10]paracyclophane [(-)-3ac].

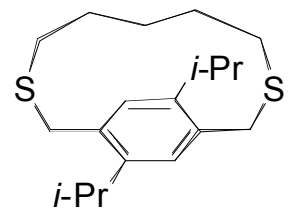

Colorless solid; Mp 53-54 ${ }^{\circ} \mathrm{C}$; $[\alpha]^{25}{ }_{\mathrm{D}}-42.4^{\circ}\left(\mathrm{CHCl}_{3}, c 1.670,37 \%\right.$ ee); IR (neat) 2959, 2925, 2866, 2360, $1458 \mathrm{~cm}^{-1}$; ${ }^{1} \mathrm{H}$ NMR $\left(\mathrm{CDCl}_{3}, 300 \mathrm{MHz}\right) \delta 6.93(\mathrm{~s}, 2 \mathrm{H}), 3.96$ (d, $J=12.9$ $\mathrm{Hz}, 2 \mathrm{H}), 3.62(\mathrm{~d}, J=12.9 \mathrm{~Hz}, 2 \mathrm{H}), 3,41$ (sept, $J=6.6 \mathrm{~Hz}, 2 \mathrm{H}) 2.55-2.46(\mathrm{~m}, 2 \mathrm{H}), 1.83$ (dt, $J=7.8,13.8 \mathrm{~Hz}, 2 \mathrm{H}), 1.41(\mathrm{~d}, J=6.6 \mathrm{~Hz}, 6 \mathrm{H}), 1.17(\mathrm{~d}, J=6.6 \mathrm{~Hz}, 6 \mathrm{H}), 0.92$ (sept, $J=6.6$ $\mathrm{Hz}, 2 \mathrm{H}$ ), 0.76 (quint, $J=6.6 \mathrm{~Hz}, 2 \mathrm{H}), 0.42$ (sept, $J=6.6 \mathrm{~Hz}, 2 \mathrm{H}) ;{ }^{13} \mathrm{C} \mathrm{NMR}\left(\mathrm{CDCl}_{3}, 75\right.$ $\mathrm{MHz}) \delta 144.0,133.7,128.9,34.9,29.9,28.2,27.7,25.4,24.5,22.3$; HRMS (EI) calcd for $\mathrm{C}_{19} \mathrm{H}_{31} \mathrm{~S}_{2}[\mathrm{M}+\mathrm{H}]^{+} 323.1867$, found 323.1844; CHIRALPAK IA, hexane $/ \mathrm{CH}_{2} \mathrm{Cl}_{2}=97: 3,0.6$ $\mathrm{mL} / \mathrm{min}$, retention times: $8.9 \mathrm{~min}$ (major isomer) and $10.3 \mathrm{~min}$ (minor isomer).

\section{(-)-11,14-Dibromo-2,8-dithia[9]paracyclophane [(-)-3ad] .}

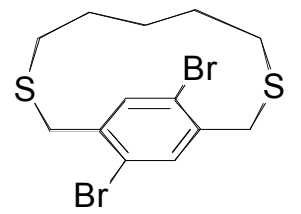

Colorless solid; Mp $107{ }^{\circ} \mathrm{C}$; $[\alpha]^{25}{ }_{\mathrm{D}}-19.6^{\circ}\left(\mathrm{CHCl}_{3}, c 1.925,27 \%\right.$ ee); IR (KBr) 2924, 1467, 1422, 1351, $1058 \mathrm{~cm}^{-1} ;{ }^{1} \mathrm{H}$ NMR $\left(\mathrm{CDCl}_{3}, 300 \mathrm{MHz}\right) \delta 7.47(\mathrm{~s}, 2 \mathrm{H}), 4.00(\mathrm{~d}, J=12.9$ $\mathrm{Hz}, 2 \mathrm{H}), 3.63(\mathrm{~d}, J=12.9 \mathrm{~Hz}, 2 \mathrm{H}), 2.50(\mathrm{dt}, J=14.1,7.5 \mathrm{~Hz}, 2 \mathrm{H}), 2.01(\mathrm{dt}, J=14.1,7.5$ 
$\mathrm{Hz}, 2 \mathrm{H}$ ), 0.96 (sept, $J=6.6 \mathrm{~Hz}, 2 \mathrm{H}$ ), 0.83 (quint, $J=6.6 \mathrm{~Hz}, 2 \mathrm{H}$ ), 0.66 (sept, $J=6.6 \mathrm{~Hz}$, $2 \mathrm{H}) ;{ }^{13} \mathrm{C} \mathrm{NMR}\left(\mathrm{CDCl}_{3}, 75 \mathrm{MHz}\right) \delta 138.1,135.7,123.3,36.5,29.8,27.9,24.3$; HRMS (EI) calcd for $\mathrm{C}_{13} \mathrm{H}_{16} \mathrm{Br}_{2} \mathrm{~S}_{2}[\mathrm{M}]^{+}$393.9060, found 393.9036; CHIRALPAK AD-H, hexane $/ 2-\mathrm{PrOH}=98: 2,1.0 \mathrm{~mL} / \mathrm{min}$, retention times: $6.8 \mathrm{~min}$ (major isomer) and $8.1 \mathrm{~min}$ (minor isomer).

\section{2,15-Dimethyl-2,9-dithia[10]paracyclophane (3ba).}

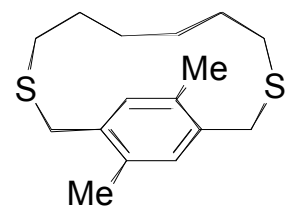

Colorless solid; Mp $69{ }^{\circ} \mathrm{C}$; IR (KBr) 2916, 2845, 1499, 1456, $690 \mathrm{~cm}^{-1}$; ${ }^{1} \mathrm{H}$ NMR $\left(\mathrm{CDCl}_{3}, 300 \mathrm{MHz}\right) \delta 7.02(\mathrm{~s}, 2 \mathrm{H}), 3.87(\mathrm{~d}, J=13.2 \mathrm{~Hz}, 2 \mathrm{H}), 3.62(\mathrm{~d}, J=13.2 \mathrm{~Hz}, 2 \mathrm{H}), 2.38$ $(\mathrm{s}, 6 \mathrm{H}), 2.21(\mathrm{t}, J=7.5 \mathrm{~Hz}, 4 \mathrm{H}), 0.99-0.74(\mathrm{~m}, 8 \mathrm{H}) ;{ }^{13} \mathrm{C} \mathrm{NMR}\left(\mathrm{CDCl}_{3}, 75 \mathrm{MHz}\right) \delta 135.0$, 134.5, 132.7, 33.9, 30.2, 28.6, 26.3, 18.8; HRMS (FAB) calcd for $\mathrm{C}_{16} \mathrm{H}_{25} \mathrm{~S}_{2}[\mathrm{M}+\mathrm{H}]^{+}$ 281.1398, found 281.1409; CHIRALCEL OD-H, hexane $/ 2-\mathrm{PrOH}=97: 3,0.7 \mathrm{~mL} / \mathrm{min}$, retention times: $31.0 \mathrm{~min}$ (major isomer) and $34.7 \mathrm{~min}$ (minor isomer).

(-)-14,17-Dimethyl-2,11-dithia[12]paracyclophane [(-)-3ca].

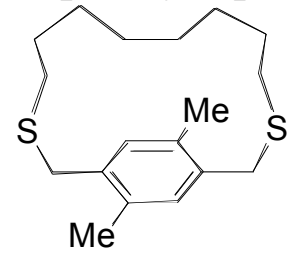

Colorless oil; $[\alpha]^{25}-14.5^{\circ}\left(\mathrm{CHCl}_{3}, c 0.755,20 \%\right.$ ee); IR (neat) 2924, 2852, 1457, 1441, $738 \mathrm{~cm}^{-1} ;{ }^{1} \mathrm{H}$ NMR $\left(\mathrm{CDCl}_{3}, 300 \mathrm{MHz}\right) \delta 6.97(\mathrm{~s}, 2 \mathrm{H}), 3.78(\mathrm{~d}, J=13.8 \mathrm{~Hz}, 2 \mathrm{H}), 3.53(\mathrm{~d}, J=$ $13.8 \mathrm{~Hz}, 2 \mathrm{H}), 2.51-2.43(\mathrm{~m}, 2 \mathrm{H}), 2.36(\mathrm{~s}, 6 \mathrm{H}), 2.36-2.26(\mathrm{~m}, 2 \mathrm{H}), 1.35-1.05(\mathrm{~m}, 8 \mathrm{H})$, $0.91-0.77(\mathrm{~m}, 4 \mathrm{H}) ;{ }^{13} \mathrm{C} \mathrm{NMR}\left(\mathrm{CDCl}_{3}, 75 \mathrm{MHz}\right) \delta 135.4,133.7,132.4,34.5,29.8,28.2,26.7$, 26.6, 18.7; HRMS (EI) calcd for $\mathrm{C}_{18} \mathrm{H}_{28} \mathrm{~S}_{2}[\mathrm{M}]^{+}$308.1632, found 308.1606; CHIRALCEL OD-H, hexane $/ 2-\mathrm{PrOH}=85: 15,1.0 \mathrm{~mL} / \mathrm{min}$, retention times: $7.5 \mathrm{~min}$ (major isomer) and $15.3 \mathrm{~min}$ (minor isomer).

\section{(-)-5,8-Dimethyl-2,11-dithia[3.3]paracyclophane [(-)-3da].}

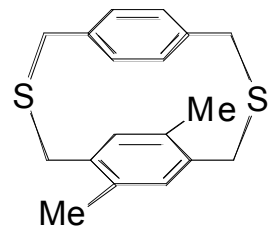

Colorless solid; Mp $182-183{ }^{\circ} \mathrm{C} ;[\alpha]_{\mathrm{D}}^{25}-23.4^{\circ}\left(\mathrm{CHCl}_{3}, c 0.285,30 \%\right.$ ee); IR (KBr) 2917, 1507, 1426, 1228, $730 \mathrm{~cm}^{-1} ;{ }^{1} \mathrm{H} \mathrm{NMR}\left(\mathrm{CDCl}_{3}, 300 \mathrm{MHz}\right) \delta 7.08(\mathrm{dd}, J=7.8,1.8 \mathrm{~Hz}$, 2H), $6.84(\mathrm{dd}, J=7.8,1.8 \mathrm{~Hz}, 2 \mathrm{H}), 6.68(\mathrm{~s}, 2 \mathrm{H}) 3.85(\mathrm{~d}, J=14.7 \mathrm{~Hz}, 2 \mathrm{H}), 3.82$ (d, $J=15.6$ 
$\mathrm{Hz}, 2 \mathrm{H}), 3.76(\mathrm{~d}, J=15.6 \mathrm{~Hz}, 2 \mathrm{H}), 3.64(\mathrm{~d}, J=14.7 \mathrm{~Hz}, 2 \mathrm{H}) 2.23(\mathrm{~s}, 6 \mathrm{H}) ;{ }^{13} \mathrm{C} \mathrm{NMR}$ $\left(\mathrm{CDCl}_{3}, 75 \mathrm{MHz}\right) \delta 135.1,133.4,133.1,132.8,129.6,127.2,37.7,35.2,19.0$; HRMS (EI) calcd for $\mathrm{C}_{18} \mathrm{H}_{20} \mathrm{~S}_{2}[\mathrm{M}]^{+} 300.1006$, found 300.0999; CHIRALCEL OD-H, hexane/2-PrOH = 97:3, $1.1 \mathrm{~mL} / \mathrm{min}$, retention times: $53.1 \mathrm{~min}$ (major isomer) and $70.7 \mathrm{~min}$ (minor isomer).

\section{(R)-(-)-5,8-Dibromo-2,11-Dithia[3.3]paracyclophane $[(R)-(-)-3 d d] .^{3}$}

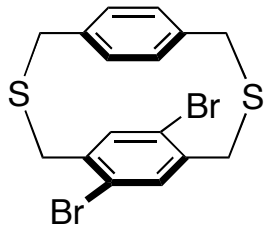

Colorless solid; Mp 223-224 ${ }^{\circ} \mathrm{C} ;[\alpha]_{\mathrm{D}}^{25}-113.7^{\circ}\left(\mathrm{CHCl}_{3}, c 0.545,98 \%\right.$ ee $) ;{ }^{1} \mathrm{H} \mathrm{NMR}$ $\left(\mathrm{CDCl}_{3}, 300 \mathrm{MHz}\right) \delta 7.18(\mathrm{~s}, 2 \mathrm{H}), 7.16(\mathrm{~d}, J=8.4 \mathrm{~Hz}, 2 \mathrm{H}), 7.01(\mathrm{~d}, J=8.4 \mathrm{~Hz}, 2 \mathrm{H}), 4.13$ $(\mathrm{d}, J=15.0 \mathrm{~Hz}, 2 \mathrm{H}), 3.86(\mathrm{~d}, J=15.0 \mathrm{~Hz}, 2 \mathrm{H}), 3.77(\mathrm{~d}, J=15.0 \mathrm{~Hz}, 2 \mathrm{H}), 3.60(\mathrm{~d}, J=15.0$ $\mathrm{Hz}, 2 \mathrm{H}) ;{ }^{13} \mathrm{C} \mathrm{NMR}\left(\mathrm{CDCl}_{3}, 75 \mathrm{MHz}\right) \delta 136.9,135.3,135.2,129.4,128.1,37.8,36.6$; CHIRALPAK IA, hexane $/ \mathrm{CH}_{2} \mathrm{Cl}_{2}=75: 25,0.7 \mathrm{~mL} / \mathrm{min}$, retention times: 9.7 min (major isomer) and $10.4 \mathrm{~min}$ (minor isomer).

\section{References}

(1) Dahrouch, M.; Jara, P.; Mendez, L.; Portilla, Y.; Abril, D.; Alfonso, G.; Chavez, I.; Manriquez, J. M.; Riviere-Baudet, M.; Riviere, P.; Castel, A.; Rouzaud, J.; Gornitzka, H. Organometallics 2001, 20, 5591.

(2) Otsubo, T.; Kohda, T.; Misumi, S. Bull. Chem. Soc. Jpn. 1980, 53, 512.

(3) Yol, K. K.; Gu, B. Y.; Won, H. D.; Yong, Y. S. Synthesis 1997, 35. 
1,4-Bis(bromomethyl)-2,5-diethylbenzene (2b)
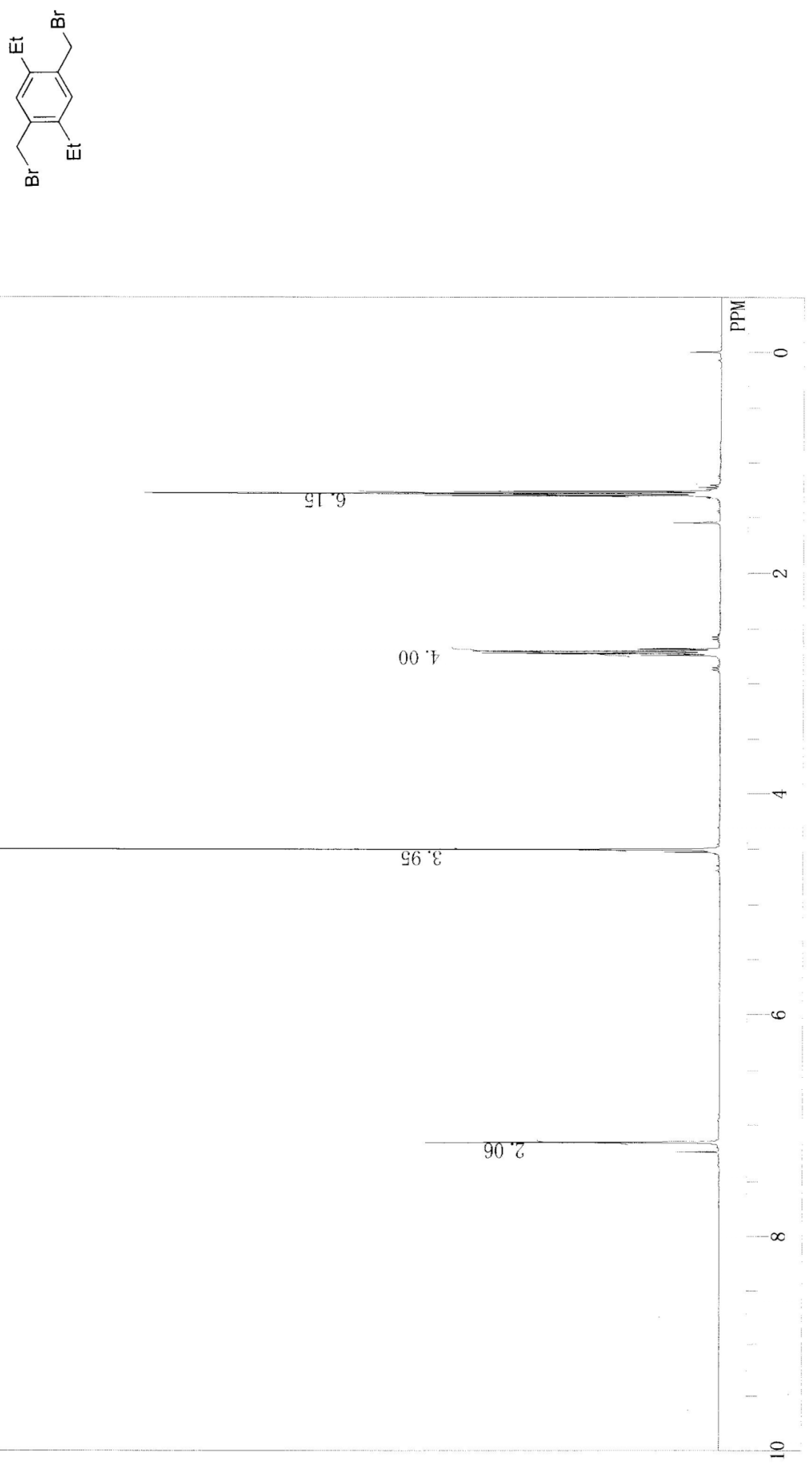
1,4-Bis(bromomethyl)-2,5-diisopropylbenzene (2c)
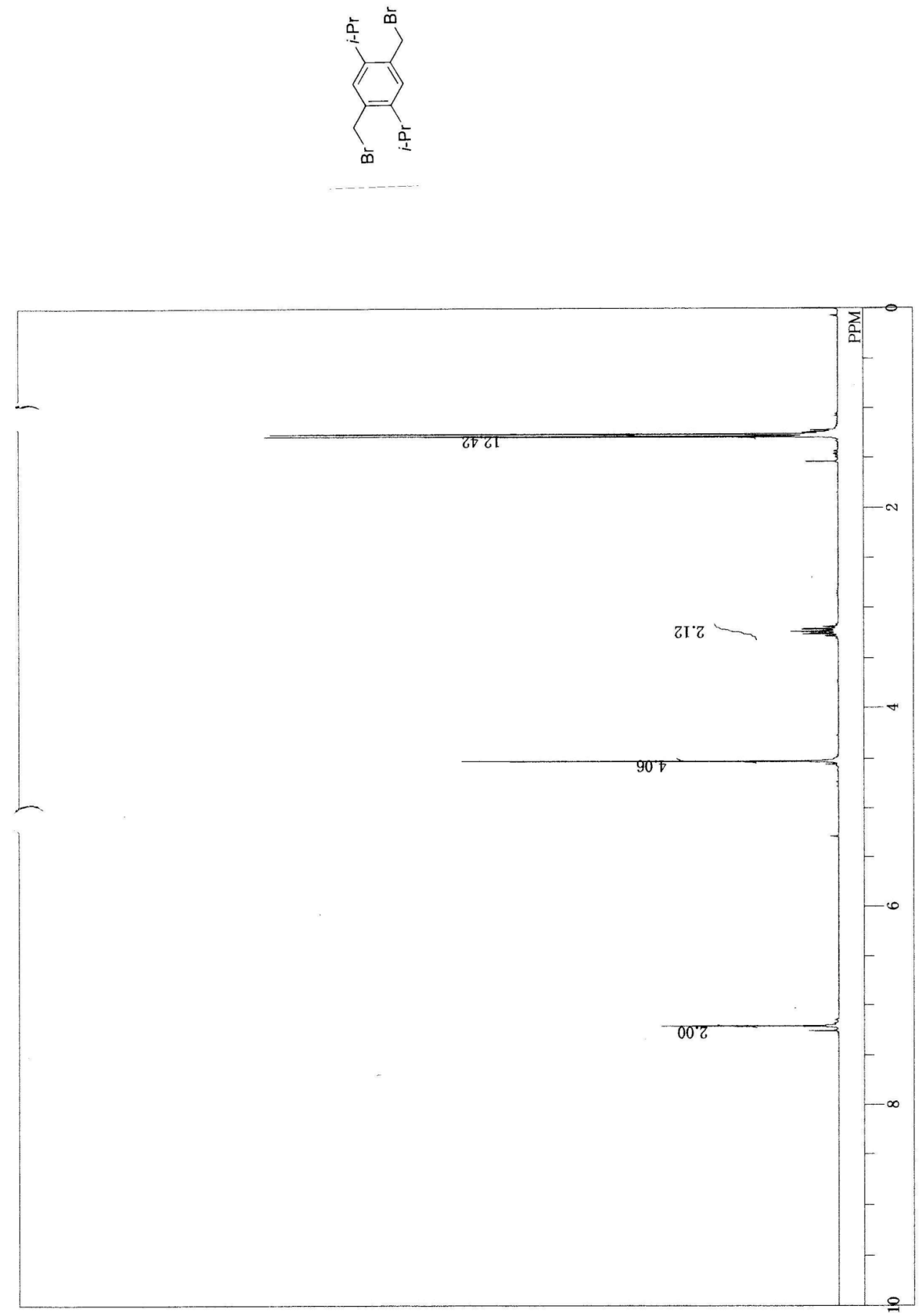
11,14-Dimethyl-2,8-dithia[9]paracyclophane (3aa)
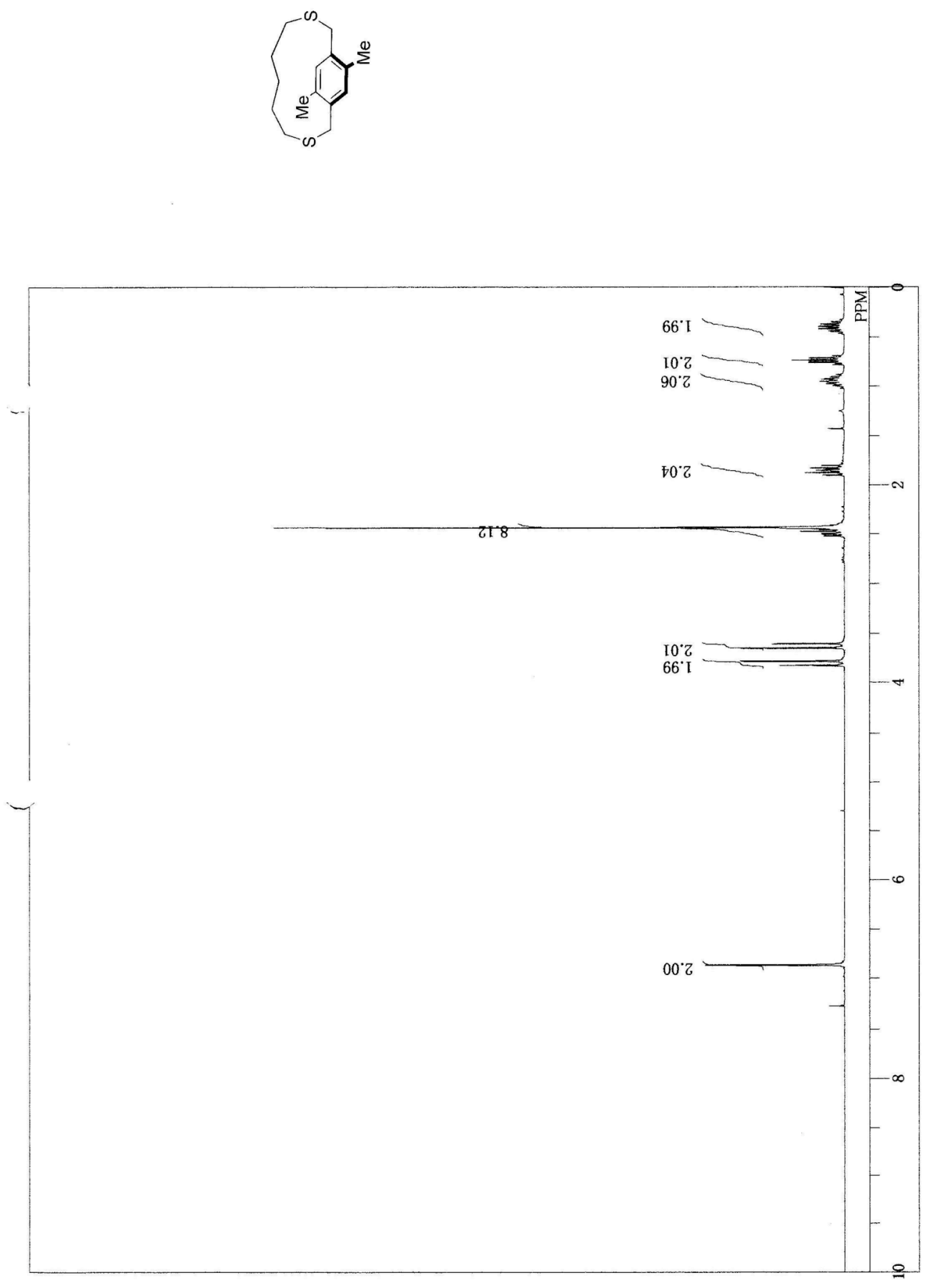
11,14-Diethyl-2,9-dithia[10]paracyclophane (3ab)
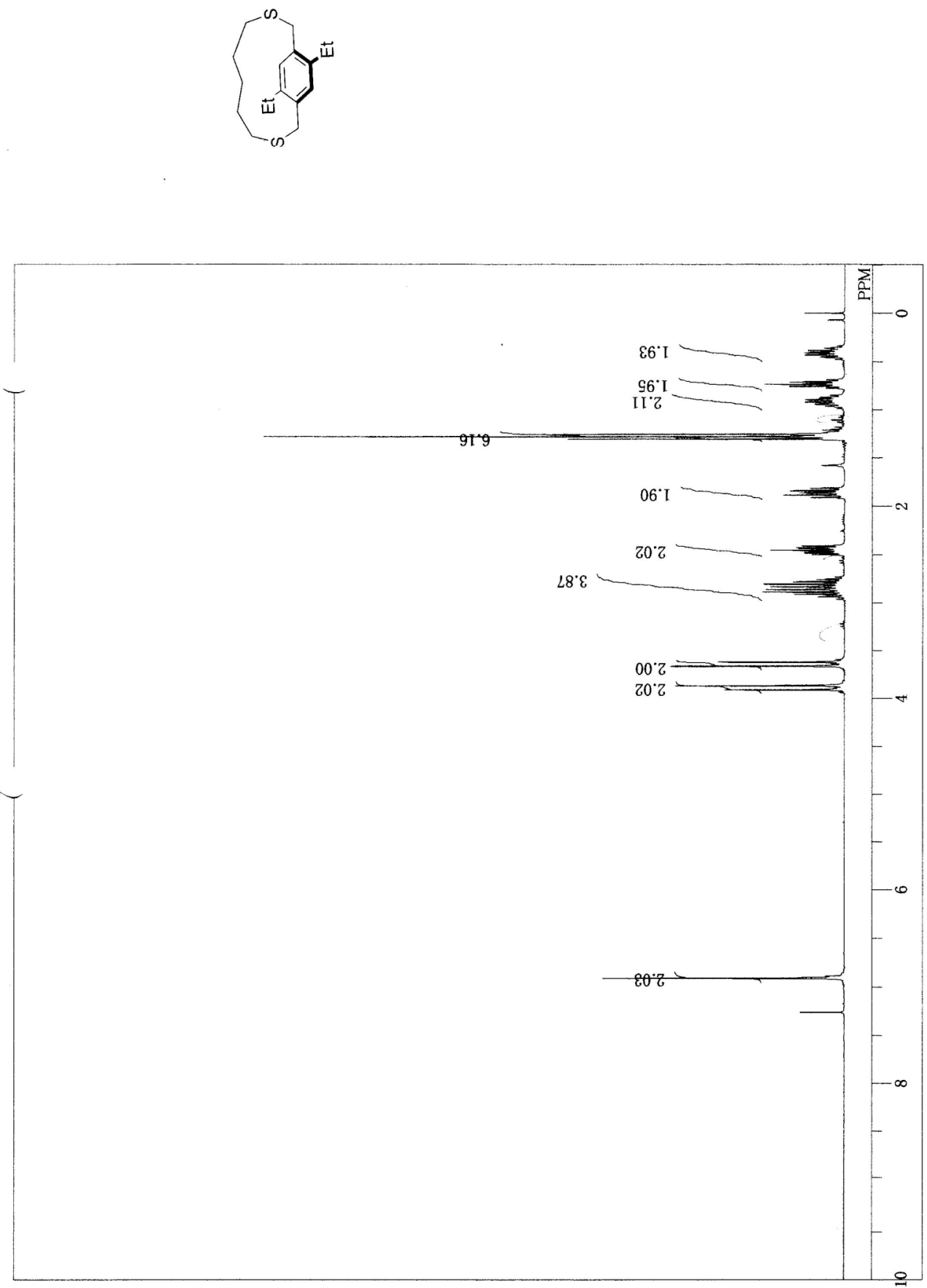
11,14-Diisopropyl-2,9-dithia[10]paracyclophane (3ac)
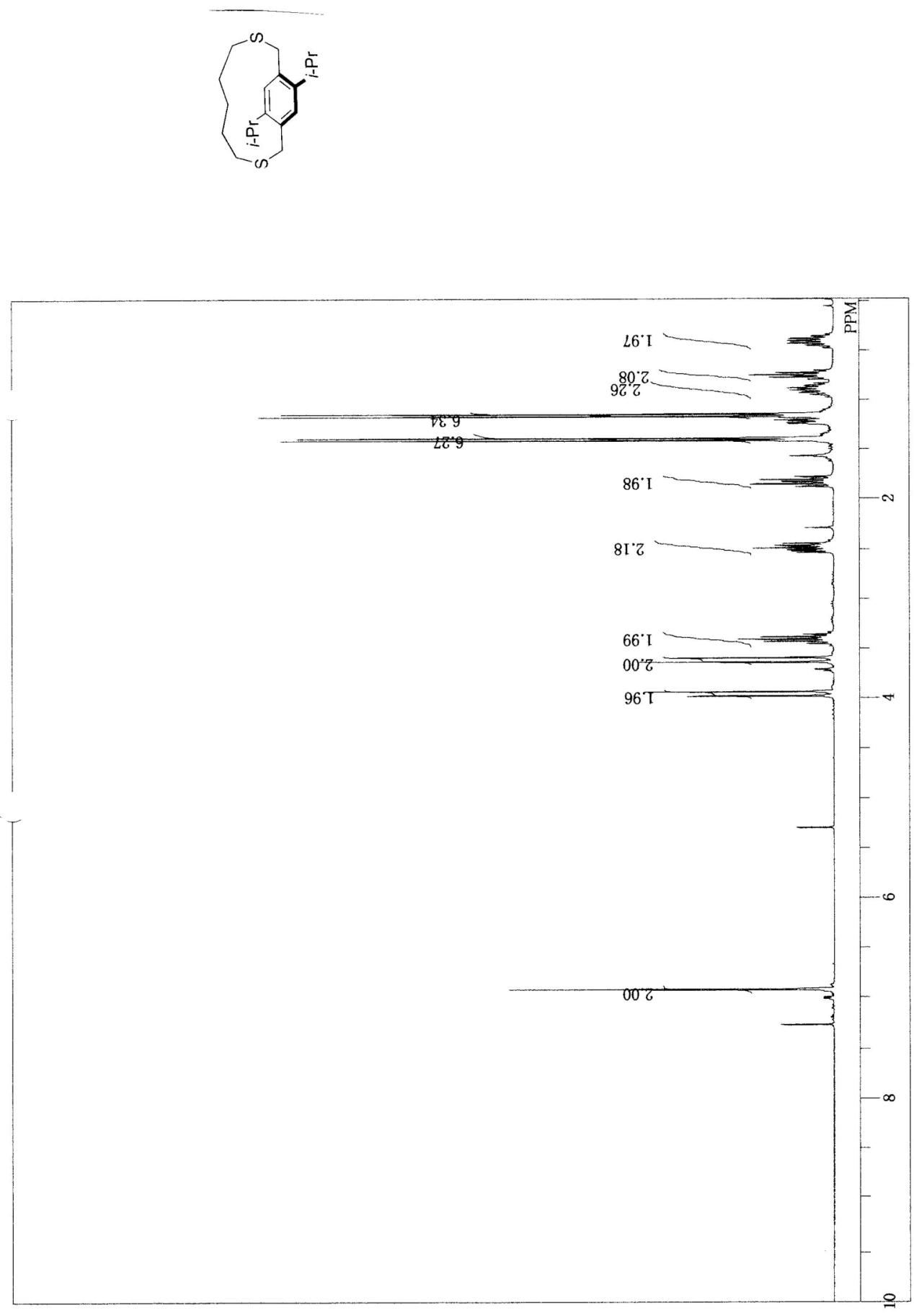


\section{1,14-Dibromo-2,8-dithia[9]paracyclophane (3ad)}
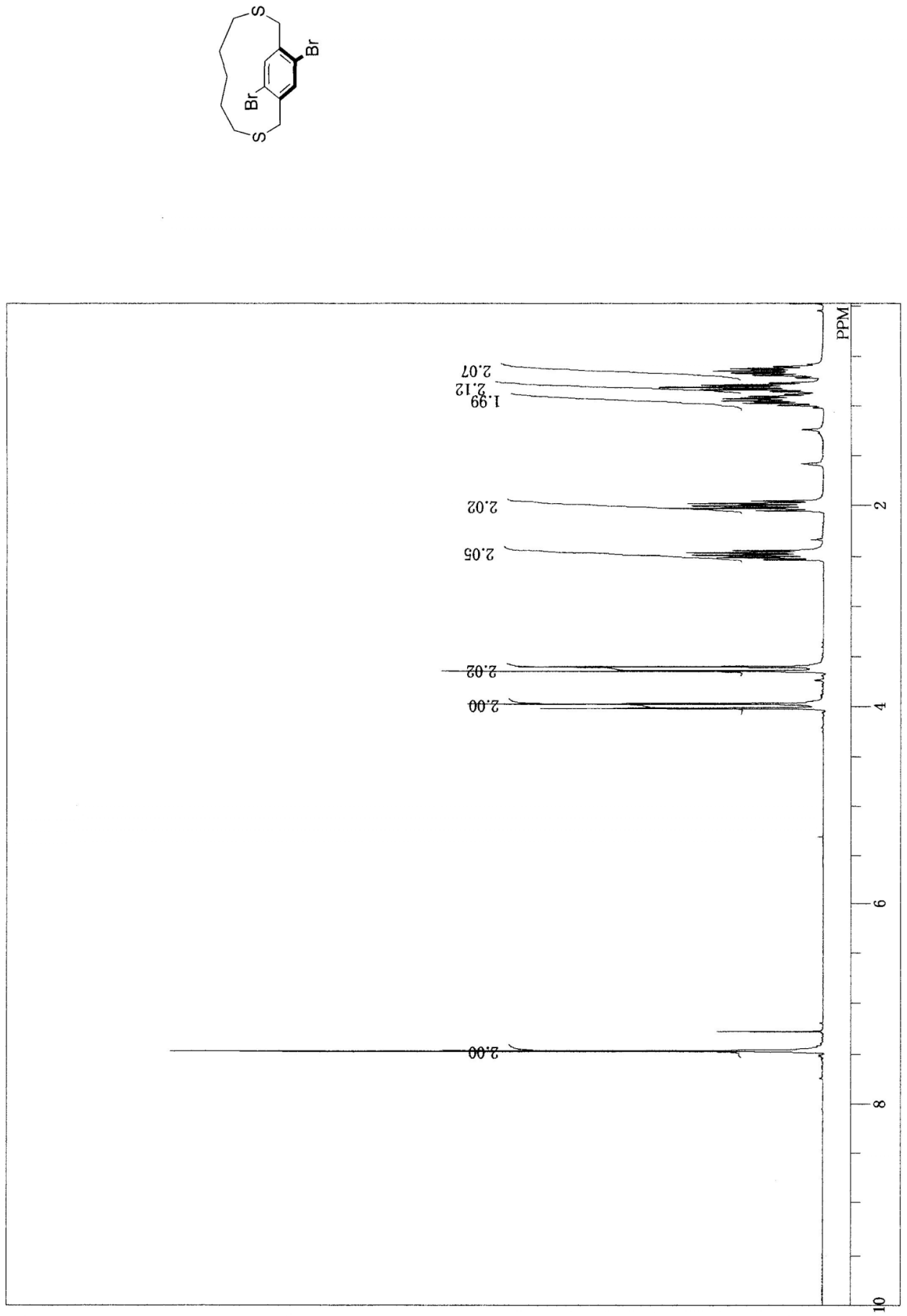
12,15-Dimethyl-2,9-dithia[10]paracyclophane (3ba)
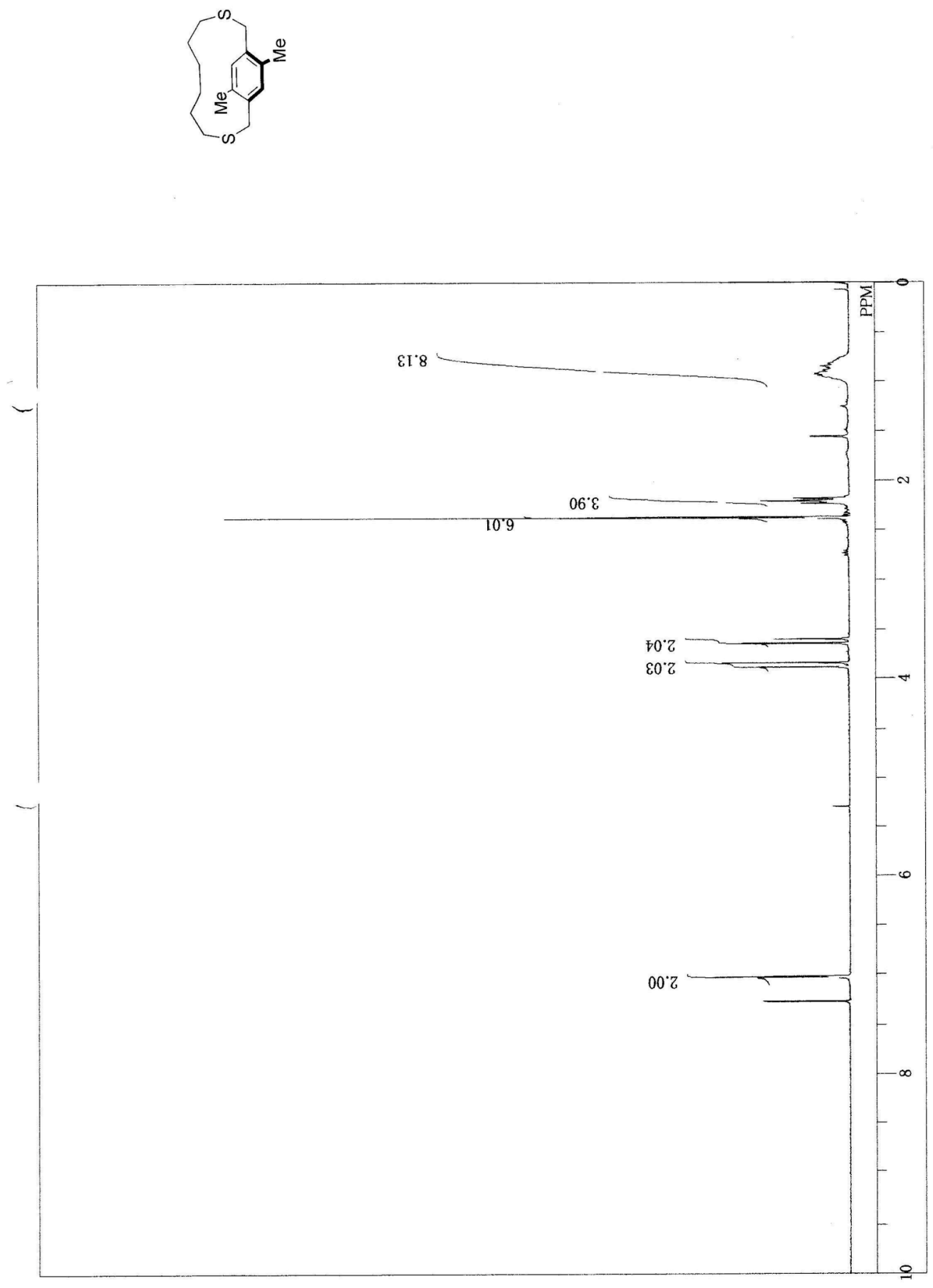


\section{4,17-Dimethyl-2,11-dithia[12]paracyclophane (3ca)}
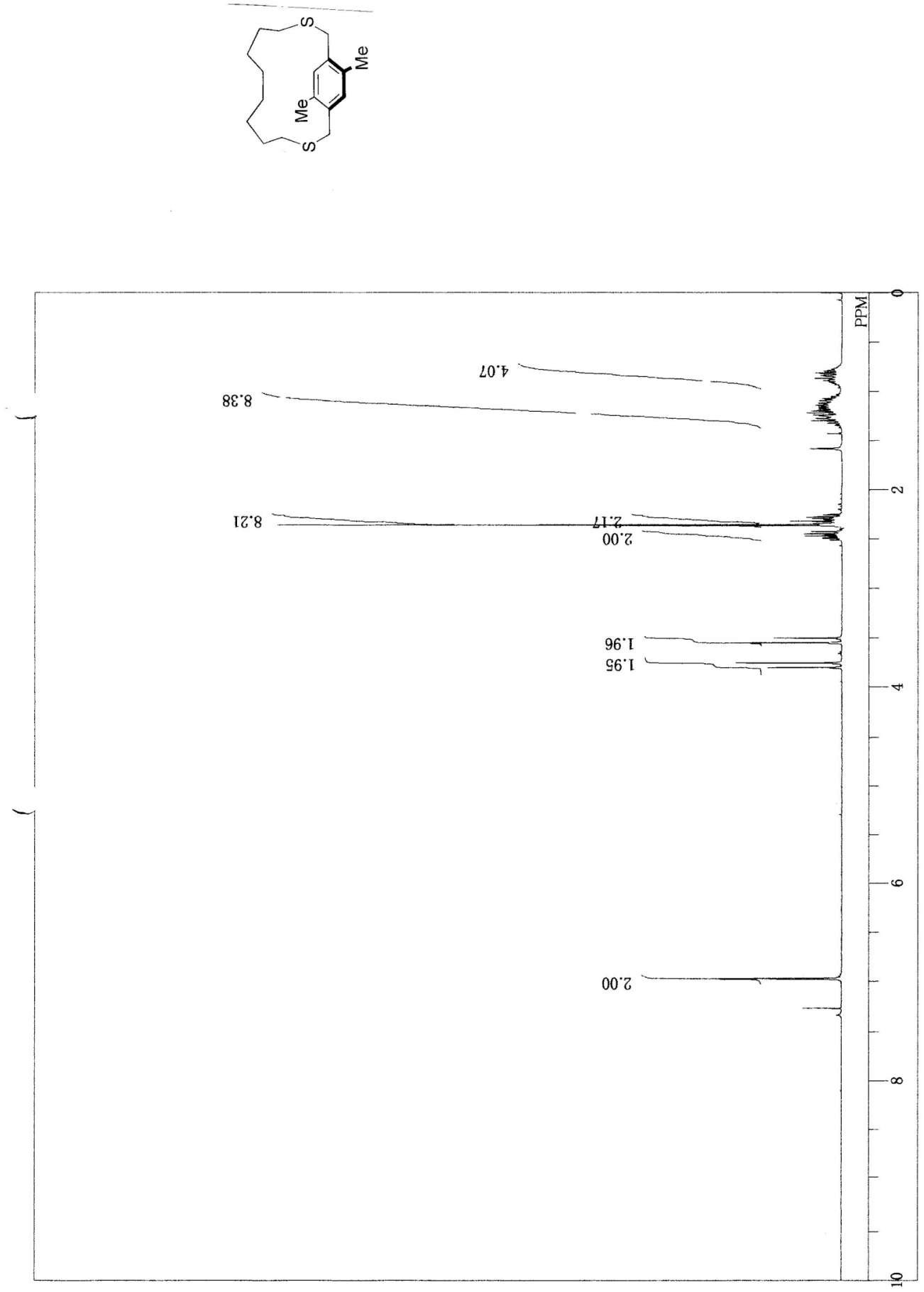
5,8-Dimethyl-2,11-dithia[3.3]paracyclophane (3da)
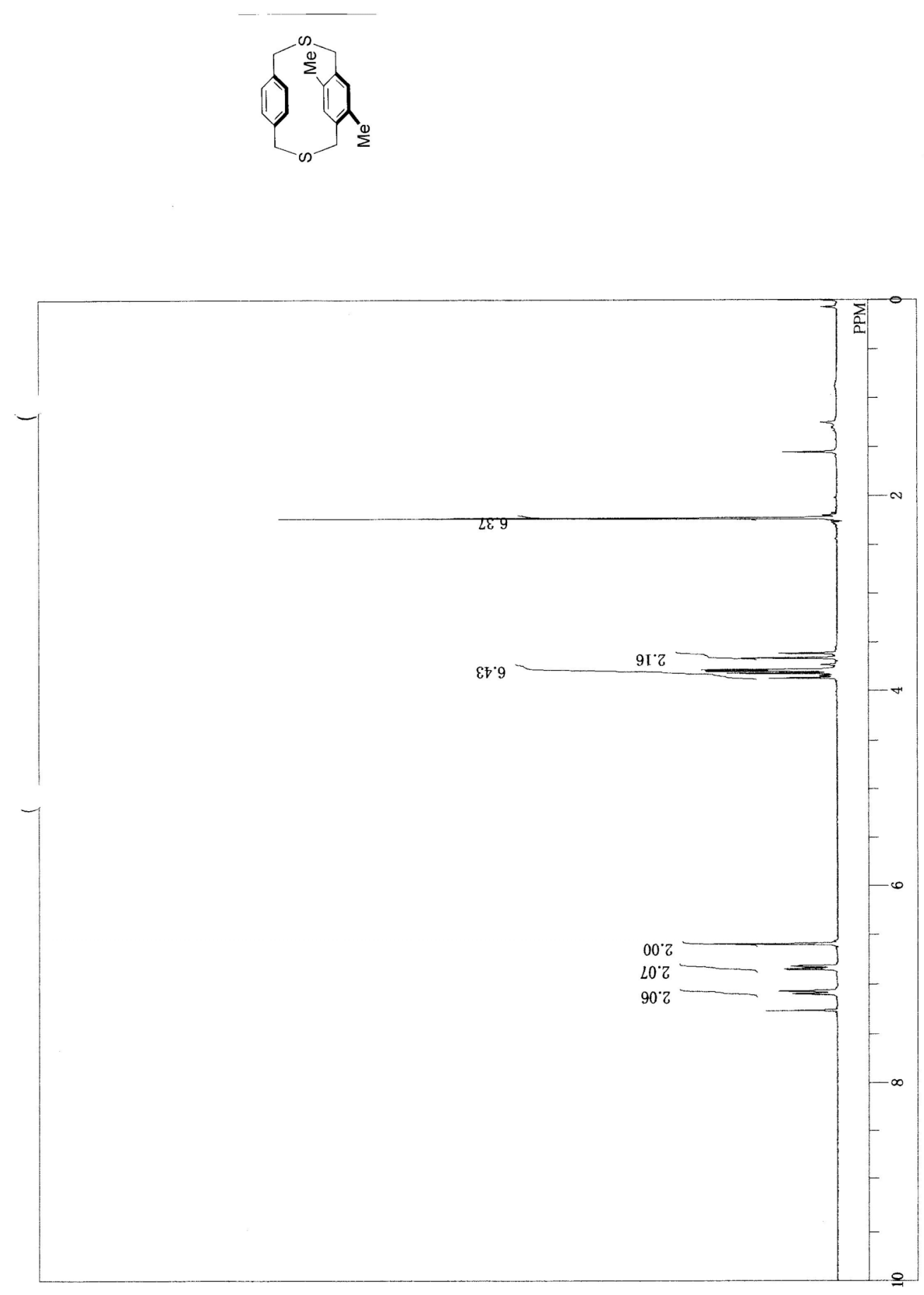
5,8-Dibromo-2,11-Dithia[3.3]paracyclophane (3dd)
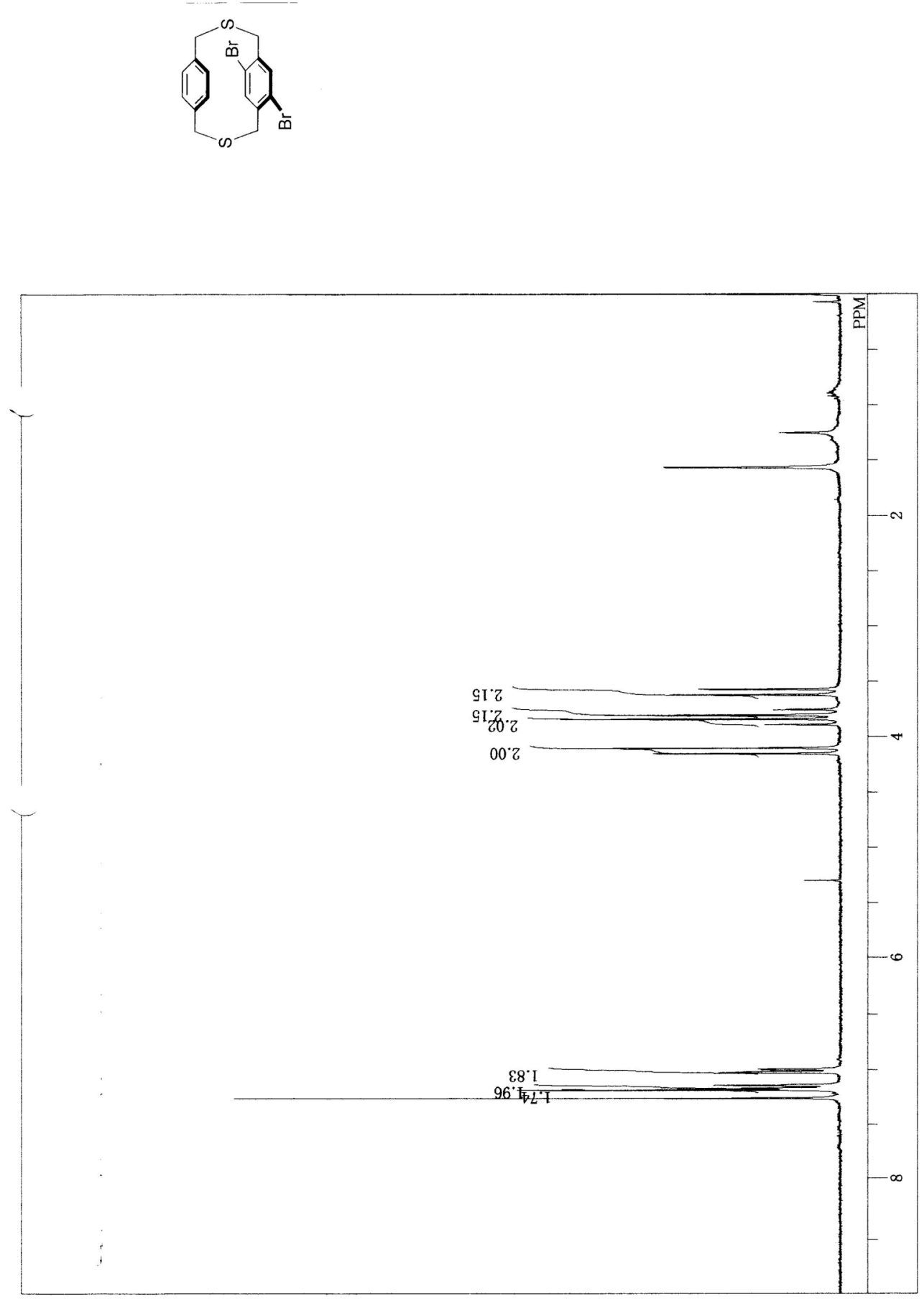